\title{
Numerical Study of Frequency-dependent Seismoelectric Coupling in Partially-saturated Porous Media
}

\author{
Ulugbek Djuraev ${ }^{1}$, Shiferaw Regassa Jufar ${ }^{1, a}$ and Pandian Vasant ${ }^{2}$ \\ ${ }^{1}$ Department of Petroleum Engineering, Universiti Teknologi Petronas, 32610 Seri Iskandar, Perak, Malaysia \\ ${ }^{2}$ Department of Fundamental \& Applied Sciences, Universiti Teknologi Petronas, 32610 Seri Iskandar, Perak, Malaysia
}

\begin{abstract}
The seismoelectric phenomenon associated with propagation of seismic waves in fluid-saturated porous media has been studied for many decades. The method has a great potential to monitor subsurface fluid saturation changes associated with production of hydrocarbons. Frequency of the seismic source has a significant impact on measurement of the seismoelectric effects. In this paper, the effects of seismic wave frequency and water saturation on the seismoelectric response of a partially-saturated porous media is studied numerically. The conversion of seismic wave to electromagnetic wave was modelled by extending the theoretically developed seismoelectric coupling coefficient equation. We assumed constant values of pore radius and zeta-potential of 80 micrometers and 48 microvolts, respectively. Our calculations of the coupling coefficient were conducted at various water saturation values in the frequency range of $10 \mathrm{kHz}$ to $150 \mathrm{kHz}$. The results show that the seismoelectric coupling is frequencydependent and decreases exponentially when frequency increases. Similar trend is seen when water saturation is varied at different frequencies. However, when water saturation is less than about 0.6 , the effect of frequency is significant. On the other hand, when the water saturation is greater than 0.6 , the coupling coefficient shows monotonous trend when water saturation is increased at constant frequency.
\end{abstract}

\section{Introduction}

The seismoelectric phenomenon has been attracting attention of many researchers all over the world. The first interest to study this phenomenon was shown the early 1940s [21]. Ever since, numerous works have been conducted to show and justify the potential of the method. Technologically, the seismoelectric method combines a high resolution of the seismic surveys and the sensitivity of the electrical methods to hydrological properties of subsurface formations $[9,23]$ that are commonly used in Oil and Gas Industry. In fact, it has been shown that the seismoelectric method can have very promising applications in various sectors of the Petroleum Engineering, especially, in Reservoir Monitoring. As such, the seismoelectric method can be applied in Reservoir Monitoring because it is able to detect subsurface fluid saturation changes. Subsequently, with sufficient knowledge of the subsurface geology, the distribution of reservoir fluids can be mapped.

As it was said earlier, the interest to explore the seismoelectric phenomena began in the twentieth century. In 1944, Frenkel investigated how streaming potentials could be generated by seismic waves in fluid-saturated porous media [7]. Thereafter, he developed a theoretical framework similar to Biot's [1] wave theory that allowed Frenkel to estimate the amount of relative fluid motion triggered by a seismic wave. Later on, he was able to calculate the magnitude of electric potentials by adopting the Helmholtz-Smoluchowski equation for streaming potentials [14].

Despite the aforementioned early breakthroughs, further investigations on the seismoelectric phenomenon were on hiatus until recently, because conducting field measurements were nearly impractical due to very low signal-to-noise ratio of the seismoelectric signals. Nevertheless, last couple of decades have been productive to deliver advances on the phenomenon thanks to theoretical breakthroughs $[11,13,14]$, a series of successful practical laboratory [24, 27, 29] and field experiments $[4,5]$; as well as to recent numerical modeling developments $[16,19,23]$.

Frequency excitation of the seismic source has a significant impact on the seismoelectric effects. Moreover, dissipation of a seismic wave travelling through a fluid-saturated porous medium is affected by the relaxation time and effect of nonlinearities that vary with fluid saturation. Therefore, this study intends to investigate the effects of seismic frequency and water saturation affecting the phenomenon via the seismoelectric coupling in two-phase saturated porous media.

\footnotetext{
a Corresponding author: shiferaw.jufar@petronas.com.my
} 


\section{Theory of seismoelectric phenomenon}

\subsection{Electrical double layer}

Seismic energy transforms into electromagnetic energy and vice versa due to the reversible electrokinetic conversion. The electrokinetic conversion occurs due to the presence of another phenomenon which is formed in a fluid-containing porous medium - electrical double layer.

As shown in Figure 1, the surface of porous medium grain, which is in contact with pore fluids, gains a chemically bound charge due to deprotonization and ion adsorption between surface and fluid reactions [18]. In a thin layer surrounding the grains, mobile counter ions tend to balance the bound charge. While the bound charge remains immobile, the transportation of counter ions is done by the flowing pore fluid. In fact, electrostatic forces and thermal diffusivity balance determine the distribution of mobile ions [20]. As a result, an electrical potential also known as the "zetapotential" is defined at the interface between immobile and counter-ions. Zeta-potential varies exponentially when it moves away from the interface. The Debye length, the corresponding characteristic decay length, is on the order of several nanometers for typical solid grainelectrolyte combinations [11]. Essentially, the adsorbed and diffuse charged layers make up the ensemble, which is referred to as the Electrical Double Layer (EDL). Hence, due to the existence of the double layer, escalation and propagation of coupled seismic and electromagnetic waves occur simultaneously.

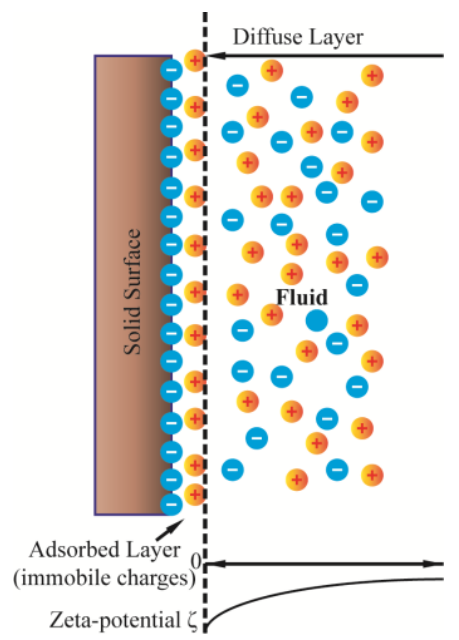

Figure 1. Electric double layer

Normally, petroleum reservoirs are predominantly water-wet. Hence the double layer is formed throughout the grain surface, and has a thickness of a few nanometers. Nonetheless, formation of the EDL on an oil-wet solid surface is also possible, though further investigation is required.

The magnitude of zeta-potential has a direct effect on the seismoelectric signals. The lower the magnitude of zeta-potential is, the weaker seismoelectric response is observed. A number of researchers have conducted experiments to measure zeta-potential for brine-saturated porous media, although the measurement procedure remains quite complex. From their experimental results they confirm that with increasing salinity, the value of zeta-potential reduces. [2, 10, 17, 22]. Moreover, they suggest that for numerical studies it is sufficient to use the empirically-derived Eq. 1 , where $C_{f}$ is brine concentration in $\mathrm{mol} / \mathrm{l}$ and $\zeta$ is zeta-potential in $\mathrm{mV}$. The equaions is presented in a general format, however, various researchers report different equation coefficients. As such, one must consider all assumptions prior using a certain model.

$$
\zeta=a \log _{10} C_{f}+b
$$

For our numerical investigation, we adopt the model presented by Vinogradov, et al. [22] that is shown below in Eq. 2. The equation remains valid for brine concentrations are up to $0.4 \mathrm{~mol} / \mathrm{l}$.

$$
\zeta=19.02 \log _{10} C_{f}-9.67
$$

\subsection{The seismoelectric effects in fluid-saturated porous medium}

When a seismic wave travels through a fluid-saturated porous medium, it induces a fluid-to-solid relative motion that leads to charge separation in the EDL. As result, an electric field is produced $[8,11,25-27,29]$. This phenomenon is referred to as "coseismic" electrical field.

A compressional wave (P-wave) travelling through a homogeneous fluid-saturated porous medium is shown in Figure 2 (left), where the wave is represented by vertical lines. There exist pressure peaks when the lines are closer together, and there exist pressure troughs when they are far apart. Hence, the counter-ions of the double layer will be transported by the fluid flow in relation to immobile, fixed charges. In this fashion, the pressure troughs are accumulated by the counter-ions $(+)$, whereby the bound charge (-) is exposed to in pressure peaks, leading to creation of the coseismic electrical field on the wavelength scale. Thus, the exact balance of the hydraulic current flow is driven by a conduction current of that electric field. Subsequently, there is not net electric current, because there is no support upon the coseismic field outside the wave $[12,13,18]$. However, the instant a seismic wave that crosses a boundary with a contrast in mechanical and/or electrical properties, it produces an electromagnetic signal referred to as the "interface response field" as shown in Fig. 5 (right).
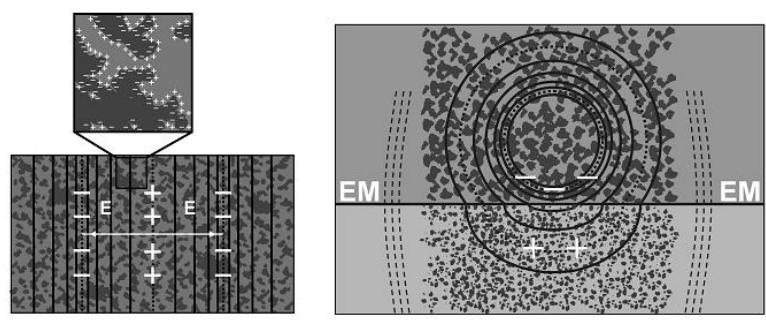

Figure 2. Seismoelectric effects: creation of the coseismic electric field (left). The interface response (electromagnetic) field is produced (right) [18] 


\subsection{Seismoelectric coupling: numerical and experimental studies}

Zhu, et al. [28] recorded conversion of seismic wave to electromagnetic wave in the laboratory borehole model at ultrasonic frequency, and showed that the seismic wave induced localized seismoelectric field and seismomagnetic field within a porous medium only. That verifies that the induced fields can only exist within the area disturbed by the travelling wave. Additionally, the they confirmed the sensitivity of the seismoelectric conversion to salinity of the pore fluid, as the amplitude of the converted electromagnetic signals decreased with increasing brine salinity [25].

Zhang, et al. [25] also point out that there is a relation between the seismoelectric coupling coefficient and rock properties. They show that the coupling coefficient increases when porosity and permeability increase too. Furthermore, Zhu, et al. [26] show that the converted electrical signal are mostly sensitive to varying permeability at a constant porosity of the formation and same acoustic source. Additionally, Warden, et al. [23] show that the seismoelectric signals are directly proportional to the pressure gradient, so the higher production rate (the higher pressure gradient) yields the more distinct seismoelectric signals.

\subsection{Frequency-dependent seismoelectric signals in fully-saturated porous media}

Zhu, et al. [29] performed a series of seismoelectric measurements in different rock samples submerged in a water tank. The recorded converted seismoelectric signals were triggered by a power hydrophone, used as an acoustic source. The source was excited with a single sine burst pulse of $100 \mathrm{kHz}$. The other hydrophone was placed near the rock samples to record acoustic field around the rock sample. Additionally, they employed a pair of electrodes to record voltage across the sample induced by the electric current due to the seismoelectric conversions. Consequently, they were able to record both acoustic disturbances and seismoelectric signals, where the conversions occurred only in the porous rock samples.

Afterwards, in order to validate that the electric signals were generated by the seismic wave exactly in the rock samples, Zhu, et al. [29] further proceeded by conducting measurements specifically over the Berea sandstone alone. Likewise, they recorded electric signals induced by the seismoelectric coupling in the frequency range $15 \mathrm{kHz}-150 \mathrm{kHz}$. As they compared the measured seismoelectric responses with theoretical calculations, they concluded that the seismoelectric coupling decreases when seismic frequency increases.

\section{Methodology}

In our study, we extended the numerical study presented by Zhu, et al. [29], where we performed calculation of the theoretical seismoelectric coupling coefficient designed for partially-saturated porous medium. Hence, we adopted the analytical coupling coefficient formula developed by Reppert, et al. [15] shown in Eq. 3 and Eq. 4. Moreover, in order to satisfy partially-saturated conditions of the variables, we also introduced additional analytical formulations developed by other researchers. As such, the density and viscosity of the fluid displayed by Eq. 5 and Eq. 6, respectively, are presented by Revil, et al. [16]. Furthermore, the formula of the dielectric constant for two-phased fluid mixture was adopted from Fabbri, et al. [6].

$$
\begin{gathered}
L=\frac{\Delta V(\omega)}{\Delta P(\omega)}=\frac{a}{2 \varepsilon_{m} \zeta \kappa} \frac{\left(\frac{2}{\kappa a} \frac{J_{1}(\kappa a)}{J_{0}(\kappa a)}-1\right)}{\left(\frac{J_{1}(\kappa a)}{J_{0}(\kappa a)}\right)} \\
\text { where } \begin{array}{c}
\kappa=\sqrt{\frac{-i \rho_{f} \omega}{\eta_{f}}} \\
\rho_{f}=S_{w} \rho_{w}+\left(1-S_{w}\right) \rho_{g} \\
\eta_{f}=\eta_{g}\left(\frac{\eta_{w}}{\eta_{g}}\right)^{S_{w}} \\
\varepsilon_{m}=\left(S_{w} \varepsilon_{w}^{\alpha}+\left(1-S_{w}\right) \varepsilon_{g}^{\alpha}\right)^{1 / \alpha}
\end{array}
\end{gathered}
$$

where $\Delta \mathrm{V}(\omega)$ and $\Delta \mathrm{P}(\omega)$ are voltage and seismic pressure at angular frequency $\omega ; J_{1}$ and $J_{0}$ are Bessel functions of first and zero orders respectively; $\zeta$ is the zeta-potential; $\varepsilon_{\mathrm{m}}$ is the dielectric constant of fluid mixture; $\mathrm{S}_{\mathrm{w}}$ is water saturation; $\eta$ and $\rho$ are viscosity and density of a fluid mixture respectively ( $\mathrm{w}$ - water, water; $\mathrm{g}$ - gas, air).

In our calculations, we assumed constant value for pore radius of $80 \mu \mathrm{m}$. Zeta-potential of $48 \mathrm{mV}$ was calculated using Eq. 2 at a constant salinity with an assumption that the EDL is not affected by changing water saturation. Values for other remaining variables were obtained from engineering tables at the temperature of $25{ }^{\circ} \mathrm{C}$. Furthermore, we performed calculations of the seismoelectric coupling at various water saturation values within the frequency range of $10 \mathrm{kHz}$ to $150 \mathrm{kHz}$.

\section{Results and Discussion}

The seismoelectric coupling was calculated for a twophased saturated porous medium. The phases considered are water and air. The calculations were performed at the range of seismic frequency and water saturation $-10 \mathrm{kHz}$ to $150 \mathrm{kHz}$ and $10 \%$ to $100 \%$, respectively. The normalized coupling coefficients against normalized frequencies are shown in Fig. 3. The coupling coefficient was calculated for every water saturation value at the given frequency range. Subsequently, we normalized the values of each coupling coefficient with respect to the largest coupling coefficient, which was obtained at the frequency of $10 \mathrm{kHz}$ for each water saturation. Furthermore, each frequency value was then normalized by the highest frequency, i.e. $150 \mathrm{kHz}$. The results show that the normalized curves at various water saturations coalesce into a single curve, indicating that the relative strength of the coupling coefficient is the same at the given frequency regardless of water saturation. Apparently, Fig. 3 illustrates the frequency-dependence 
of the coupling coefficient, where the normalized curves at each water saturation values decreases exponentially when normalized frequency increases. The decline trend of the coupling can be divided into two regions depending on the normalized frequency. When the normalized frequency $\omega_{n}$ is low, i.e. less than about 0.4 $(60 \mathrm{kHz})$, the coupling coefficient decreases fast. On the other hand, when the normalized frequency $\omega_{n}$ is high, i.e. greater than about 0.4 , the coupling coefficient decreases slowly.

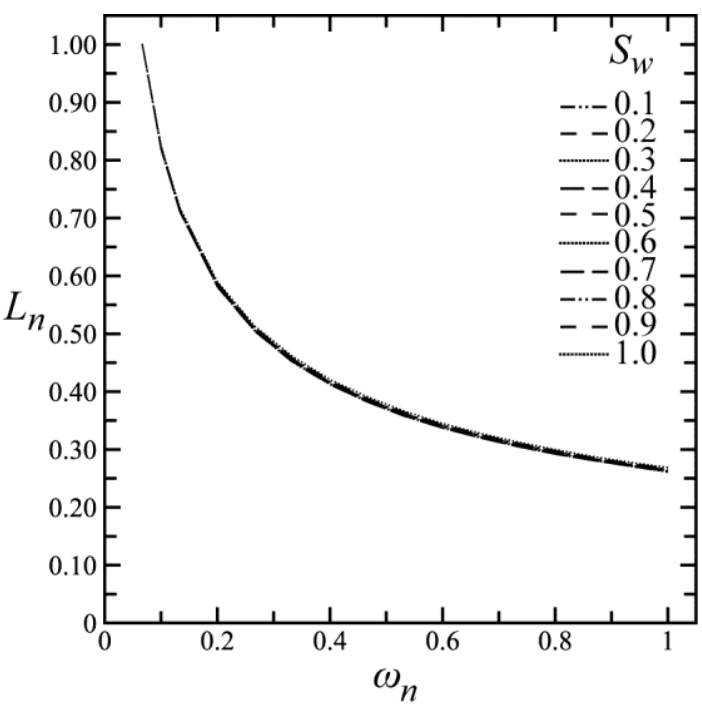

Figure 3. Normalized seismoelectric coupling $\left(L_{n}\right)$ as a function of normalized seismic frequency $\left(\omega_{n}\right)$

Apparently, at high frequency, the seismoelectric conversion is less efficient due to interference and nonlinearities and as such, the coupling coefficient is low at higher frequencies. Figure 4 shows variation of the normalized coupling coefficient with water saturation at various seismic frequencies. As it can be observed from the figure, the frequency-dependence of the coupling appears to be significant in the lower frequency range, i.e. less than $60 \mathrm{kHz}$. However, frequencies greater than 60 $\mathrm{kHz}$ does not affect the coupling coefficient that much. Moreover, Fig. 3 and Fig. 4 imply that the seismoelectric coupling curves would exhibit identical trends, irrespective of water saturation.

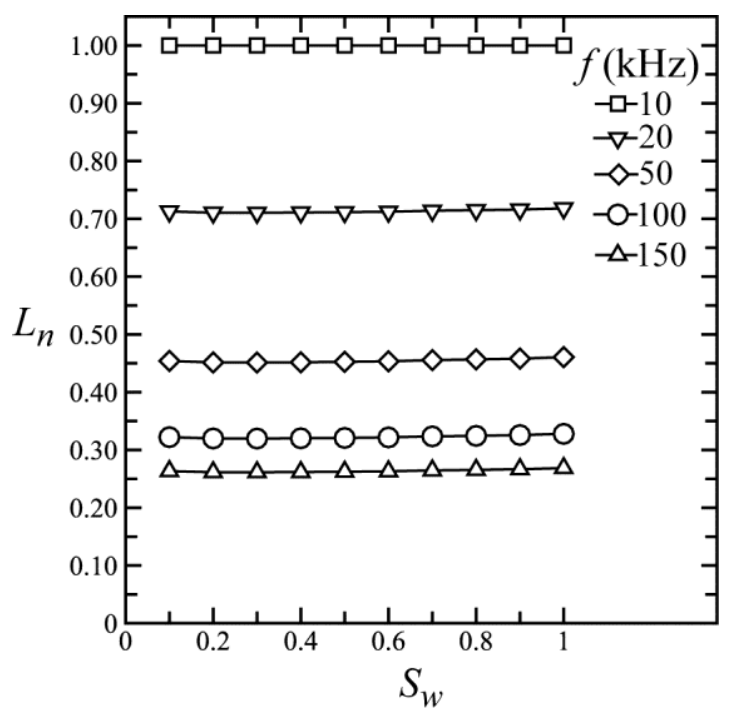

Figure 4. Normalized seismoelectric $\left(\boldsymbol{L}_{\boldsymbol{n}}\right)$ as a function of water saturation $\left(\boldsymbol{S}_{w}\right)$

Figure 5 shows effect of water saturation on the seismoelectric coupling at various frequencies. Brine concentration was fixed at $0.01 \mathrm{~mol} / \mathrm{l}$. When water saturation is 0.1 , the magnitude of the coupling coefficient reduces drastically by $380 \%$, i.e. from 0.19 $\mathrm{nV} / \mathrm{Pa}$ to $0.05 \mathrm{nV} / \mathrm{Pa}$, when frequency increases from 10 $\mathrm{kHz}$ to $150 \mathrm{kHz}$. Although subsequent reduction in the coupling coefficient is seen when water saturation is fixed and frequency increases, the decline rate lessens when water saturation increases from 0.1 to 0.6 . When water saturation is greater than 0.6 , the rate of reduction of the coupling coefficient remains almost constant as frequency increases at fixed water saturation suggesting that frequency has negligible effect on the coupling coefficient at high water saturation.

In a porous medium saturated with water and gas, the seismoelectric coupling is rather enhanced at low water saturations. Our results obtained from investigating the effect of water saturation on the coupling coefficient show consistence with experimental and theoretical findings obtained by Bordes, et al. [3]. Additionally, our results are in full agreement with findings of $\mathrm{Zhu}$, et al. [29], where they show frequency-dependence of the coupling in fluid-saturated porous media. 


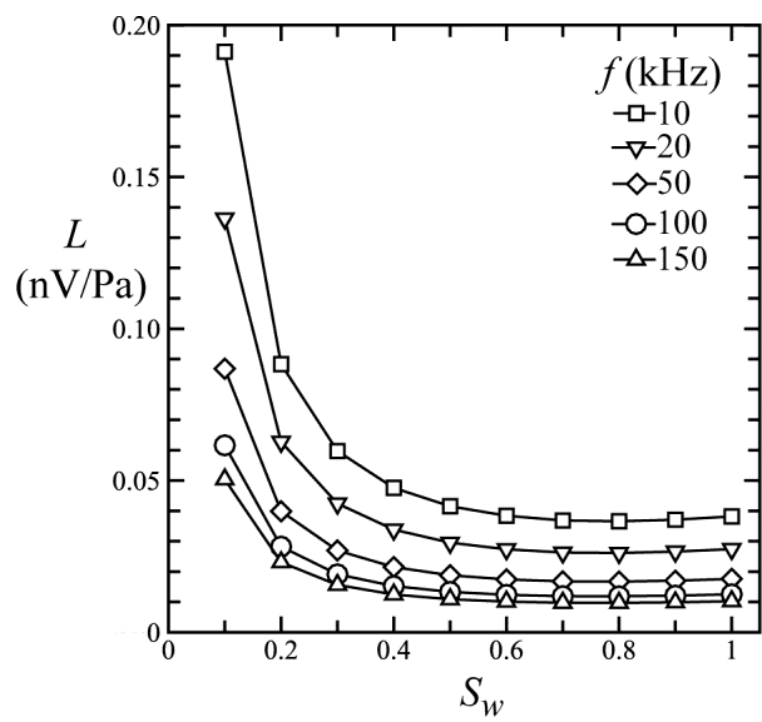

Figure 5. Seismoelectric coupling as a function of water saturation $\left(\boldsymbol{S}_{w}\right)$ and frequency $(\boldsymbol{f})$

\section{Conclusion}

In this paper, we extended the calculation of theoretical seismoelectric coupling coefficient to partially-saturated conditions, whereby the ratio was a function of seismic frequency and water saturation. Our results suggest that regardless of fully- or partially-water saturated conditions, the seismoelectric coupling coefficient reduces when frequency increases. At lower water saturation, i.e. less than 0.6 , the magnitude of the coupling coefficient significantly drops when frequency increases. On the contrary, the magnitude of the coupling coefficient remains rather constant when water saturation increases at constant frequency. When water saturation is greater than about 0.6 , regardless of frequency the rate of decline of the coupling coefficient remains almost constant. The results observed in our study are consistent with experimental and numerical findings of other researchers.

\section{Acknowledgements}

The authors express their gratitude to UTP for providing financial support under cost-centre No. 0153AB-DA5. We thank the editor and the referees for valuable comments and their time.

\section{References}

1. M.A. Biot. "Theory of propagation of elastic waves in a fluid-saturated porous solid. II. Higher frequency range," the Journal of the Acoustical Society of America Vol. 28, No. 2, (1956), pp. 179-191.

2. A. Boleve, A. Crespy, A. Revil, F. Janod, and J.-L. Mattiuzzo. "Streaming potentials of granular media: Influence of the Dukhin and Reynolds numbers," Journal of Geophysical Research: Solid Earth Vol. 112, No. B8, (2007).

3. C. Bordes, P. Sénéchal, J. Barrìère, D. Brito, E. Normandin, and D. Jougnot. "Impact of water saturation on seismoelectric transfer functions: a laboratory study of coseismic phenomenon," Geophysical Journal International Vol. 200, No. 3, (2015), pp. 1317-1335.

4. J.C. Dupuis, K.E. Butler, and A.W. Kepic. "Seismoelectric imaging of the vadose zone of a sand aquifer," Geophysics Vol. 72, No. 6, (2007), pp. A81-A85.

5. J.C. Dupuis, K.E. Butler, A.W. Kepic, and B.D. Harris. "Anatomy of a seismoelectric conversion: Measurements and conceptual modeling in boreholes penetrating a sandy aquifer," Journal of Geophysical Research Vol. 114, No. B10, (2009).

doi: 10.1029/2008jb005939

6. A. Fabbri, T. Fen-Chong, and O. Coussy. "Dielectric capacity, liquid water content, and pore structure of thawing-freezing materials," Cold Regions Science and Technology Vol. 44, No. 1, (2006), pp. 52-66.

7. J. Frenkel. "On the theory of seismic and seismoelectric phenomena in a moist soil," J. Phys. J. Phys. (Soviet) Vol. 8, No. 4, (1944), pp. 230-241.

8. N. Grobbe, J. Hunziker, and E. Slob. "Seismoelectric wave propagation modeling for typical laboratory configurations: A numerical validation," (2014), pp. 2072-2077. doi: 10.1190/segam2014-0689.1

9. S.S. Haines, S.R. Pride, S.L. Klemperer, and B. Biondi. "Seismoelectric imaging of shallow targets," Geophysics Vol. 72, No. 2, (2007), pp. G9-G20.

10. M. Jaafar, J. Vinogradov, and M. Jackson. "Measurement of streaming potential coupling coefficient in sandstones saturated with high salinity $\mathrm{NaCl}$ brine," Geophysical Research Letters Vol. 36, No. 21, (2009).

11. S.R. Pride. "Governing equations for the coupled electromagnetics and acoustics of porous media," Physical Review B Vol. 50, No. 21, (1994), p. 15678

12. S.R. Pride, and S. Garambois. "The role of Biot slow waves in electroseismic wave phenomena," The Journal of the Acoustical Society of America Vol. 111, No. 2, (2002), pp. 697-706.

13. S.R. Pride, and M.W. Haartsen. "Electroseismic wave properties," The Journal of the Acoustical Society of America Vol. 100, No. 3, (1996), pp. 1301-1315.

14. S.R. Pride, and F. Morgan. "Electrokinetic dissipation induced by seismic waves," Geophysics Vol. 56, No. 7, (1991), pp. 914-925.

15. P.M. Reppert, and F.D. Morgan. "Frequencydependent electroosmosis," Journal of Colloid and interface Science Vol. 254, No. 2, (2002), pp. 372383.

16. A. Revil, G. Barnier, M. Karaoulis, P. Sava, A. Jardani, and B. Kulessa. "Seismoelectric coupling in unsaturated porous media: theory, petrophysics, and saturation front localization using an electroacoustic approach," Geophysical Journal International, (2013), p. ggt440.

17. A. Revil, P. Pezard, and P. Glover. "Streaming potential in porous media: 1 . Theory of the zeta 
potential," Journal of Geophysical Research: Solid Earth Vol. 104, No. B9, (1999), pp. 20021-20031.

18. M.D. Schakel. Coupled seismic and electromagnetic wave propagation: TU Delft, Delft University of Technology, (2011).

19. M.D. Schakel, Z. Zhu, and E.C. Slob. "Fullwaveform model and measurements of electromagnetic to seismic conversion," 2011 SEG Annual Meeting. Society of Exploration Geophysicists, (2011).

20. J.J. Sheng. "Foams and Their Applications in Enhanced Oil Recovery," Enhanced Oil Recovery Field Cases Elsevier, (2013), pp. 251-276.

21. R. Thompson. "The seismic electric effect," Geophysics Vol. 1, No. 3, (1936), pp. 327-335.

22. J. Vinogradov, M. Jaafar, and M. Jackson. "Measurement of streaming potential coupling coefficient in sandstones saturated with natural and artificial brines at high salinity," Journal of Geophysical Research: Solid Earth Vol. 115, No. B12, (2010).

23. S. Warden, S. Garambois, L. Jouniaux, D. Brito, P. Sailhac, and C. Bordes. "Seismoelectric wave propagation numerical modelling in partially saturated materials," Geophysical Journal International Vol. 194, No. 3, (2013), pp. 1498-1513.

doi: $10.1093 /$ gji/ggt198

24. X. Zhan, Z. Zhu, and M.N. Toksoz. "Quantitative DC And High Frequency AC Seismoelectric Measurement On Berea Sandstone," 2011 SEG Annual Meeting. Society of Exploration Geophysicists, (2011).

25. Y. Zhang, and J. Zhang. "An Experimental Study of Seismoelectric Conversion in Model Borehole," 2003 SEG Annual Meeting. Society of Exploration Geophysicists, (2003).

26. Z. Zhu, S. Chi, X. Zhan, and M.N. Toksöz. "Theoretical and experimental studies of seismoelectric conversions in boreholes," Commun. Comput. Phys Vol. 3, No. 1, (2008), pp. 109-120.

27. Z. Zhu, and M.N. Toksöz. "Experimental Studies Of Electrokinetic Conversions In A Fluid-Saturated Porous Medium." Massachusetts Institute of Technology. Earth Resources Laboratory, (1996).

28. Z. Zhu, and M.N. Toksöz. "Crosshole seismoelectric measurements in borehole models with fractures," The 7th International Symposium on Recent Advances in Exploration Geophysics (RAEG 2003). (2003).

29. Z. Zhu, M.N. Toksöz, and D.R. Burns. "Electroseismic and seismoelectric measurements of rock samples in a water tank," Geophysics Vol. 73, No. 5, (2008), pp. E153-E164. 